\title{
USING EXPERT OPINION IN HEALTH TECHNOLOGY ASSESSMENT: A GUIDELINE REVIEW
}

\author{
Theresa Hunger \\ Petra Schnell-Inderst \\ Narine Sahakyan \\ Department of Public Health, Health Services Research and Health Technology Assessment, \\ UMIT- University for Health Sciences, Medical Informatics and Technology \\ theresa.hunger@umit.at \\ Division of Public Health Decision Modeling, Health Technology Assessment and Health \\ Economics, ONCOTYROL - Center for Personalized Cancer Medicine
}

\begin{abstract}
Uwe Siebert
Department of Public Health, Health Services Research and Health Technology Assessment, UMIT- University for Health Sciences, Medical Informatics and Technology

Division of Public Health Decision Modeling, Health Technology Assessment and Health Economics, ONCOTYROL - Center for Personalized Cancer Medicine

Department of Health Policy and Management, Harvard T.H. Chan School of Public Health Institute for Technology Assessment and Department of Radiology, Massachusetts General Hospital, Harvard Medical School
\end{abstract}

Objectives: External experts can be consulted at different stages of an HTA. When using vague information sources, it is particularly important to plan, analyze, and report the information processing in a standardized and transparent way. Our objective was to search and analyze recommendations regarding where and how to include expert data in HTA. Methods: We performed a systematic database search and screened the Internet pages of seventy-seven HTA organizations for guidelines, recommendations, and methods papers that address the inclusion of experts in HTA. Relevant documents were downloaded, and information was extracted in a standard form. Results were merged in tables and narrative evidence synthesis.

Results: From twenty-two HTA organizations, we included forty-two documents that consider the use of expert opinion in HTA. Nearly all documents mention experts in the step of preparation of the evidence report. Six documents address their role for priority setting of topics, fifteen for scoping, twelve for the appraisal of evidence and results, another twelve documents mention experts when considering the dissemination of HTA results.

During the assessment step, experts are most often asked to amend the literature search or to provide expertise for special data analyses. Another issue for external experts is to appraise the HTA results and refer them back to a clinical and social context. Little is reported on methods of expert elicitation when their input substitutes study data.

Conclusions: Despite existing recommendations on the use of expert opinion in HTA, common standards for elicitation are scarce in HTA guidelines.

Keywords: Health technology assessment, Expert opinion, Expert elicitation, Expert data

The systematic evaluation of health related technologies within a health technology assessment (HTA) is a multistage process including not only the assessment of clinical effectiveness (1-4). The applicable jurisdiction and decision process determine the conduct of a HTA. Typically, it starts with the priority setting of assessment topics. Next is the scoping, where a specific research question is selected and explicated on the basis of predefined criteria. The assessment in a narrower sense, the creating of the assessment report, starts after these steps and ideally considers clinical benefits and harms, ethical, social, legal, and organizational aspects, and, if applicable, costeffectiveness of the procedure using the best available evidence. After evaluating the scientific evidence, the results are appraised. Finally, the results are disseminated and periodically updated. Sometimes implementation of results is supported.

The present work was exclusively funded by the Tiroler Wissenschaftsfonds, grant number GZ: UNI - 4040/1009.
Each step in the HTA process uses different sources of information and involves different groups of persons. For the assessment step, the best available scientific evidence should be regarded. Randomized controlled trials (RCT) — or even better, synthesis of several trials - provide the most reliable evidence for estimates of the clinical effectiveness of therapies (5); however, they are not always available and do not serve all research questions. Expert opinion, on the other hand, is situated on the lowest level of the hierarchy of evidence. Nevertheless, technical experts are consulted when empirical data are not available or for example for questions of practice patterns, preferences, procurement processes, or economic data $(6 ; 7)$.

Sullivan and Payne (8) diagnosed an increasing need for using expert opinion in economic models due to the growing number of assessments of interventions without data from randomized controlled trials, namely diagnostics. Despite the wide application of expert data in modeling studies, it has been argued that expert opinion was applied inconsistently and its use reported insufficiently (9). 
When information of a low evidence level is used in HTA, it is even more important to systematically plan and document the data collection and analysis and to report it in a transparent way. The aim of the present study was to review the current state of recommendations from general HTA guidelines and handbooks regarding the involvement of experts and expert opinion in different steps of the HTA process.

\section{METHODS}

\section{Terminology}

We refer to "experts" as external persons with a special knowledge or expertise in a relevant field who are not routine members of the HTA team. With the term "expert opinion," we summarize all contributions experts can make to the HTA process. This can be quantitative and qualitative data, experience, or value judgments.

\section{Literature Search}

We performed a Web search for guidelines and methods papers considering expert involvement in HTA on the Internet pages of seventy-seven international HTA agencies and organizations. We selected all agencies participating in the European network for HTA (EUnetHTA) during Joint Action 1 and all members of the International Network of Agencies for HTA (INAHTA) and searched their Web sites by means of search fields or hand search in the sections "tools," "methods," "resources," "publications," etc.. All screened institutions are listed in the Supplementary Table 1. As an additional source of publications, we screened the bibliography of the recent EUnetHTA guideline on methods for health economic evaluations, which is based on a review of methodological guidelines developed by the members of EUnetHTA (10).

Finally, we consulted the database "Pharmacoeconomic Guidelines Around The World" on the ISPOR Web site (www. ispor.org/PEguidelines/index.asp). The initial search from 2011 was updated in March 2015. Documents were retrieved and read in full-text if they were available in English or German language, contained the search terms "expert, specialist, professional," and if they had a general methodological focus. They were excluded from further analysis if they did not address our research question regarding expert involvement in the HTA process. We complemented our search by an electronic database search in Medline and Embase for further guidelines in German and English language through August 2011. The syntax is provided in the Supplementary Tables 2 and 3. The Internet search and download of documents was performed by one author (T.H.) and included documents reviewed by a second author (N.S. or P.S-I.). Results from the database search were screened in a two-step process (first title and abstract, then full text) by one reviewer (T.H.).

\section{Information Synthesis}

For the document analysis, relevant information was extracted according to a predefined scheme by one author (T.H) and reviewed by a second independent author (N.S. or P.S-I.).

We defined five steps of the HTA process and applied this structure to all evidence tables. First, we identified at what HTA step each document mentioned expert involvement and how detailed this information was. We also extracted available information about who is considered an expert and how they are identified and selected by the HTA organizations. The nature and content of experts' contributions was summarized and illustrated by statements from the documents. We listed the various elicitation methods by HTA step.

\section{RESULIS}

\section{Existing Guidelines}

From the HTA agency Web sites, the electronic database search, the bibliography screening and the ISPOR guideline database, we retrieved 120 documents for full-text screening (see Supplementary Figure 1). We finally included forty-two documents from twenty-two institutions that address the involvement of expert opinion in the HTA process (Table 1). None of the included documents stemmed from the electronic database search.

The twenty-two organizations are very heterogeneous in the manner they integrate experts in HTA and in the extent they report about it in their handbooks. The National Institute for Health and Care Excellence (NICE), for example, gives a detailed description of its process of expert involvement (11-14). Several organizations have more than one guidance document and expert involvement sometimes differs with respect to the area which is covered by the guideline, for example, economic evaluation or systematic literature review.

\section{Selection of Experts}

The guideline documents generally define experts as persons who are not routine members of the HTA team $(11 ; 15-17)$. However, there are different conceptions about who is regarded an expert that should be involved in the HTA (Table 2). First, there are content experts who have particular knowledge of the technology under investigation. Content experts can come from different areas of expertise (18), primarily healthcare professions (19), but also ethicists are recruited as content experts (20). Second, there are methods experts, whose expertise are analytical techniques for evidence evaluation or synthesis $(15 ; 21)$. Methods experts are also mentioned for quality assurance $(3 ; 22 ; 23)$. A third group that contributes to HTA are patients, patient representatives, dependents, and caregivers, because they provide expert information about the perspective of the persons concerned $(11 ; 13 ; 14)$. Beyond that, some 
Table 1. Consideration of Expert Involvement at Different HTA Steps According to HTA Handbooks

\begin{tabular}{|c|c|c|c|c|c|c|c|}
\hline Institution (reference) & Year & Country & Prioritization & Scoping & Assessment & Appraisal & Dissemination \\
\hline AAZ (21) & 2011 & Croatia & & + & + & + & + \\
\hline AHTAPol (41) & 2009 & Poland & & & + & + & \\
\hline ASERNIP-S (17) & 2003 & Australia & & & + & & \\
\hline CADTH (36) & 2006 & Canada & & & ++ & & \\
\hline CADTH (37) & 2009 & Canada & & & + & & \\
\hline CRD (15) & 2009 & UK & & & + & & + \\
\hline CVZ (40) & 2006 & Netherlands & & & ++ & & \\
\hline CVZ (29) & 2007 & Netherlands & & & + & + & \\
\hline DACEHTA (22) & 2007 & Denmark & & + & + & & \\
\hline DAHTA@DIMDI (48) & 2008 & Germany & + & & & & + \\
\hline EUnetHTA (54) & 2013 & Europe & & & + & & \\
\hline EuroScan (27) & 2014 & Europe & + & & ++ & & + \\
\hline GÖG (30) & 2012 & Austria & + & + & + & & \\
\hline GÖG (32) & 2010 & Austria & & + & ++ & & + \\
\hline HAS, general (47) & 2007 & France & & & + & + & + \\
\hline HAS, rapid assessment (18) & 2007 & France & & & + & & \\
\hline HAS (33) & 2012 & France & & & + & & \\
\hline HAS (20) & 2013 & France & & & +++ & & \\
\hline HIQA (39) & 2010 & Ireland & & & + & & \\
\hline HIQA, budget impact (28) & 2014 & Ireland & & & + & & \\
\hline HIQA, clinical effectiveness (43) & 2014 & Ireland & & & + & & \\
\hline HIQA, economic evaluation (49) & 2014 & Ireland & & & ++ & & \\
\hline HIQA, stakeholder engagement (31) & 2014 & Ireland & & & +++ & & \\
\hline INAHTA (44) & 2005 & International & & + & & & \\
\hline INFARMED (42) & 1998 & Portugal & & & ++ & & + \\
\hline IQWiG (16) & 2015 & Germany & & + & ++ & + & + \\
\hline KCE (46) & 2008 & Belgium & & & + & & \\
\hline KCE, cost-based pricing (35) & 2012 & Belgium & & & + & & \\
\hline KCE, economic evaluation (26) & 2012 & Belgium & & & +++ & & \\
\hline LBI (23) & 2007 & Austria & & + & ++ & & + \\
\hline LBI (3) & 2008 & Austria & & + & ++ & & + \\
\hline MoH (38) & 2012 & Malaysia & & & + & & \\
\hline NICE (12) & 2007 & UK & & + & ++ & + & \\
\hline NICE (11) & 2009 & UK & + & + & + & + & \\
\hline NICE, diagnostics (55) & 2011 & UK & & + & + & & \\
\hline NICE, methods (19) & 2011 & UK & & + & + & + & \\
\hline NICE, process (25) & 2011 & UK & & + & ++ & + & \\
\hline NICE (13) & 2013 & UK & & +++ & +++ & +++ & \\
\hline NICE (14) & 2014 & UK & + & + & +++ & +++ & + \\
\hline OHTAC (24) & 2009 & Canada & & & + & & \\
\hline ОНТAC (45) & 2010 & Canada & & & ++ & + & \\
\hline PHARMAC (34) & 2012 & New Zealand & + & & + & & + \\
\hline Sum & & & 6 & 15 & 40 & 12 & 12 \\
\hline$\%$ & & & 14 & 36 & 95 & 29 & 29 \\
\hline
\end{tabular}

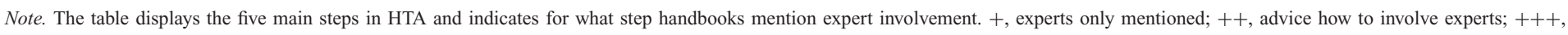
detailed description of the expert involvement.

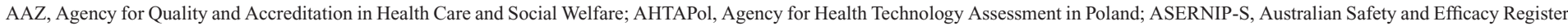

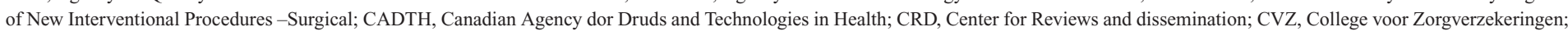

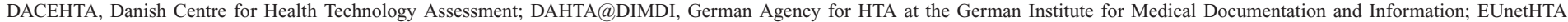

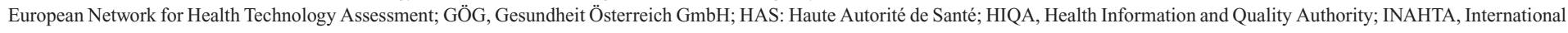

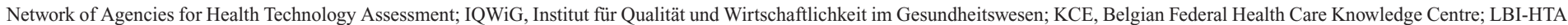

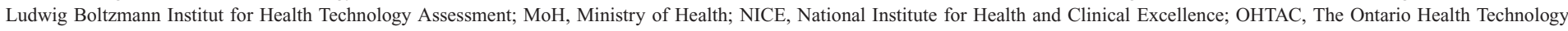
Advisory Committee; PHARMAC, Pharmaceutical Management Agency. 
Hunger et al.

Table 2. Different Kinds of Experts Are Considered at Different Steps in the HTA Process

\begin{tabular}{ll} 
Step in the HTA process & \\
\hline $\begin{array}{l}\text { Priority setting } \\
\text { Scoping }\end{array}$ & $\begin{array}{l}\text { Stakeholder, professional expert } \\
\text { Content expert, patient expert, caregiver }\end{array}$ \\
Data collection & Content expert, clinical expert, methods expert, industry representative, patient representative, study authors, advisory group \\
Data analysis/synthesis & $\begin{array}{c}\text { Clinical expert, content expert, advisory surgeon, statistical expert, industry representative, patient representative, expert panel, } \\
\text { consensus panel, invited members } \\
\text { Patient representative, personal contacts, international (research) networks }\end{array}$ \\
Appraisal & Peer reviewer \\
Dissemination and reporting
\end{tabular}

Table 3. Nature of expert contribution to HTA

Step in the HTA process

Contributions of experts

Suggest and select topics
Briority setting
Bcoping
technology; role of technology in the health care system; provide views on rules for starting and
stopping use of technology; identify requirements for implementation of guidance on the
technology;
Background on disease: information on personal impact of a disease
Other: development of research question; check whether there is enough evidence available; identify
variations in clinical practice
Literature search: construct search strategy; identification of relevant literature; selection of
bibliographic databases; information about unpublished evidence; identification of relevant journals;
check literature list;
Literature selection: appraise study quality, refine how to answer QuADAS score
Decision-analytic modeling
Model structure: determine usual or recommended care, actual dosing, and resource use; determine
transferability of resource use between countries; select comparators; perform quality assessment
of models;
Model parameterization: provide model data input, effectiveness data input, translate efficacy data
into effectiveness, value outcomes, establish parameter values, define plausible ranges for values;
Sensitivity analysis: determine extent of parameter variability, determine weights for age and health
states, determine discount rates, model validation, determine degree of precision of cost
assessment, handle data limitations;
Other analyses: ordinal data, mixed data scales, complex mixed treatment comparisons, Bayesian
methods, individual patient data meta-analysis; identify effect modifiers; assess process of patient
management; identify ethical dilemmas arising from development and use of the technology
Provide a view of the technology in current practice with insights not available from literature; provide
perspective on reality; provide advice that complements findings from research;
Determine patient relevant outcomes; information about users' needs;
'teach back': evaluate information from literature
Review draft report; help to develop a lay version of the guidance; information on ethical and societal
values; inform feasibility of adoption of HTA results; stimulate inter-peer discussion

Note. Statements from HTA handbooks about the kind of contribution that experts provide are listed for each HTA step. The assessment step is separated into data collection on the one hand and analysis and synthesis on the other. 
institutions refer to stakeholders (e.g., providers, manufacturers, payers) as separate expert group (24).

For the selection of content experts, NICE for example, contacts professional bodies, follows the recommendations of individuals who have formerly served as advisors, and performs literature searches to identify experts $(11 ; 25)$. Usually, two clinical experts, two patient experts and two commissioning experts are selected for NICE's Technology Appraisal Program (14). A list of advisors is published on the agency's Web site. Patient commentators are recruited by means of hospitals. The German Institute for Quality and Efficiency in Health Care (Institut für Qualität und Wirtschaftlichkeit im Gesundheitswesen, IQWIG) publishes a call for proposals containing qualification requirements on its Web site (16).

Only eight institutions give details of the expert recruitment process $(3 ; 11 ; 16 ; 20 ; 21 ; 26-28)$. Most of them state that experts are selected according to criteria reflecting expertise in methods and/or content and that they must declare potential conflicts of interests. The Dutch Health Care Insurance Board explicitly states that experts that were consulted by the registration holder of the technology under investigation will not be involved (29). We found reference for the inclusion of either individual experts or panels with varying numbers of members. Some agencies recommend the inclusion of more than one expert to get a range of views (27;30). The size of expert panels is between 5 and 20 individuals in the Irish guideline for stakeholder engagement (31).

\section{Expert's Contribution}

Table 1 provides an overview of all included documents and indicates the HTA step, where they mention involvement of experts. We found that all but two guidelines $(n=40$; 95 percent) involve experts in the assessment of the technology in one way or another. Fifteen reports (36 percent) involve experts in the scoping process, that is, twenty of twenty-two institutions. Nearly thirty percent $(n=12)$ of the reports consider experts for the dissemination of the HTA report which also includes quality assurance and review of the final HTA report. Another twelve reports mention experts for appraisal of evidence and six (14 percent) note their role in the prioritization of assessment topics.

In general, we distinguish between experts giving advice regarding the conduct of an HTA and providing background information on the technology under study, and those who represent an additional data source for the analysis of clinical and cost-effectiveness, thus substituting empirical data. Table 3 provides a summary of the information from handbooks about the nature of input institutions seek from experts at each HTA step.

To prioritize possible topics for HTA reports, experts and stakeholders are asked to identify and weight relevant topics for assessment. During scoping, experts usually help framing the research question $(3 ; 11 ; 23)$, denote issues where evidence is missing $(22 ; 29)$, and indicate points to be considered in the assessment. Particularly, they give background information about the technology under investigation and affected disorders. NICE, for example, involves clinical and patient experts in the committee's entire upfront discussions about the technology that is assessed (11).

In the preparation of the assessment report, a major contribution of external advisors is the support of the literature search. They are frequently consulted to locate relevant studies $(3 ; 15 ; 23 ; 30 ; 32)$ and provide information about ongoing and unpublished research $(12 ; 27)$. After literature has been collected, experts discuss results of study inclusion and appraise the quality of included studies $(3 ; 15 ; 23 ; 30)$. The Centre for Reviews and Dissemination (CRD) (15) recommends involving different kinds of experts in every step of the literature search.

Another crucial area for expert opinion during the assessment step is the evidence synthesis and analysis. In our review, HTA guidelines agree that expert data is low-grade evidence, but many suggest expert advice, for example, for healtheconomic and decision-analytic modeling, if other evidence is deficient $(16 ; 26 ; 33-35)$. The majority of recommendations for data analysis refer to decision-analytic and health economic modeling. In this context, most method papers emphasize to state in the report which assumptions or data which populate the model are based on expert opinion. For model parameters based on expert opinion, the Canadian Agency for Drugs and Technologies in Health (CADTH) recommends sensitivity analysis (36;37).

The expert contribution to the model structure includes, among others, assessing the process of patient management (16), selecting comparators $(36 ; 38)$, determining usual or recommended care as well as actual dosing (36) and resource consumption $(15 ; 16 ; 22 ; 35 ; 36 ; 39)$, judging the transferability of resource data between countries, or general validation of the model $(3 ; 16 ; 34 ; 40)$ (Table 3$)$. To parameterize and populate the model with data, experts are asked to assess the variability of input parameters $(34 ; 36 ; 41)$, value quality of life data $(36 ; 42)$, determine weights for age and health states (42), determine discount rates, and ascertain the required precision of cost assessment (16). Regarding the analysis techniques, CRD explicitly recommends seeking additional input from statisticians with special expertise for Bayesian methods, complex indirect treatment comparisons, mixed data scales, and meta-analysis of individual patient data (15). Particularly for Bayesian methods, experts are asked to provide prior information for the analyses (43).

Experts' contribution is also of matter in the field of ethical and social aspects of a health technology $(20 ; 44 ; 45)$. More general questions to experts during the assessment step focus on the substantiation of existing knowledge $(11 ; 46)$ and the implementation into clinical practice (45). Finally, we found 
recommendations to seek expert review to assure the quality of the HTA report $(3 ; 16 ; 22 ; 23 ; 32 ; 42)$. Some guidelines request to report all external experts involved in the assessment $(13 ; 14 ; 25)$, a justification and the method of elicitation $(20 ; 36 ; 41)$.

Expert opinion for critical appraisal is recommended in HTA manuals but only limited details are provided. In summary, the experts are asked to evaluate and complement the information gathered from research and to contextualize the scientific evidence within the clinical and social context of the use of a technology $(11 ; 16 ; 41 ; 45)$.

The contribution to the dissemination step ranges from a general review of the HTA report $(13 ; 21 ; 27)$ to the detailed answering of questions regarding "readability, the proposals made, and any future action to be taken regarding the development or distribution of the technology" by means of checklists (47). The National Authority of Medicines and Health Products (INFARMED, I.P.) guideline (42) explicitly calls on experts to disseminate the HTA results and stimulate the inter-peer discussion, while the German Agency for Health Technology Assessment (DAHTA) at the German Institute of Medical Documentation and Information (DIMDI) (48) only mentions general support of experts in stimulating information exchange with other agencies.

\section{Elicitation Methods}

Most documents give the advice that, if expert opinion is a source of information, it should be reported how it was obtained, but the majority do not specify this any further. HIQA states that "where expert opinion is used, it should be sought in a manner which minimizes bias and the process should be documented in sufficient detail" (49). The advice for expert elicitation (Table 4) is mainly restricted to vague instructions: "seek input" (36), "consult" (15), "the methods should be described" (15). NICE and HAS's ethical aspects guideline describe the methods used for information retrieval from experts in more detail $(11 ; 13 ; 14 ; 20)$. Also the Belgian Health Care Knowledge Centre KCE (26) lists specific reporting items for the expert elicitation. They include potential blinding of participants to the study's purpose, remunerations, background information provided, the medium used to collect the opinions, questions asked, use of iteration in collation, approach used to present variability in opinions, and approach used to finalize the estimates from different experts. Altogether, established methods of social research are recommended (16), namely focus groups, interviews, written surveys (16;30), or consensus panels (42) (e.g., Delphi method) (30) and also triangulation of methods (30). The Ontario Health Technology Advisory Committee states in its handbook that systematic methods for elicitation of societal and ethical values are being developed (45).

\section{DISCUSSION}

While many public HTA institutions consider experts in the HTA process, we found little advice on how they do this. Most of the screened methods guidelines at least mention expert involvement. In the core step of an HTA, the assessment of benefits and risks of a technology, experts may represent a data source if empirical studies are not available. For this essential task, some fundamental advice is given in the agency handbooks, but the recommendations usually remain vague or just generally demand a description of the expert elicitation.

To our knowledge, this is the first systematic overview of the consideration of expert opinion in HTA guidance documents. Our research is limited by the document search on organizations' Web sites. This way of information retrieval is not completely systematic, and we did not apply quality criteria to the included documents. The organizations we screened might have additional internal standards which we did not include. Even the organizations that did not publish any guidelines on their Web sites might still have practical guidance documents for the preparation of HTA reports. It may have been beneficial to contact the agencies and ask for such internal handbooks, but this was not possible in the time frame of the study. We limited our analysis to documents issued by HTA agencies and similar institutions, although there are other producers of HTA as well.

The reason for this was that we want to describe the methods of institutions that have some kind of authority, for example, because they are government appointed, and their guidelines have a binding character, also for third parties. This may possibly bias the methods we found to more conventional and conservative approaches. More experimental methods may be used by other producers of HTA, for example, academic research groups or manufacturers doing internal assessments or early HTA. Certainly, further research should also consider academic institutions. Another limitation it that even though we screened international organizations we included only reports in English and German language. Particularly when considering national HTA agencies, there are often no translations of their documents available. The results from the electronic database search were screened by only one reviewer, but as this search was only meant to complement the Web search, we believe this to be sufficiently comprehensive.

Our analysis reflects the methodological standards of organizations that perform or commission HTA, but we have not validated their theoretic guidelines against how they are actually implemented in HTA studies. It is possible, that in practice, the approaches to expert data are different. For example, HTA doers might follow published methods for expert elicitation coming from different sources and research areas.

When clear guidelines on selection of experts, elicitation methods, and implementation of expert data in an HTA are lacking, this might distort the findings based on this study. The formalized elicitation of numerical data (point estimates or distributions of parameters) should be judged differently from 
Table 4. Different Methods of Expert Elicitation Reported in HTA Handbooks

Step in the HTA process

Elicitation method

\begin{tabular}{ll}
\hline Prioritization & Communication, involvement \\
Scoping & Provide written evidence; provide written personal view; provide oral commentary; involvement \\
Data collection & Interview, focus group, Delphi method, questionnaire, methods triangulation, seek advice \\
Data analysis/synthesis & Consultation, communication; inter-peer discussion; involvement, contact, comment; survey \\
Appraisal & (Semi-structured) interview; group interview, focus group; written questionnaire, opinion poll, survey \\
Dissemination and reporting & Colloquial forms of evidence; experience; systematic methods are being developed \\
\hline
\end{tabular}

using expert opinion, for example, to obtain background information on the technology use. A recent methodology review of expert judgement on distributions in model-based HTA concludes that "reports of expert elicitation are insufficient in detail and this impacts on the perceived usability of expert-elicitated probability distributions" (50).

Where expert data replaces empirical data, it should be treated with the same rigor as data from trials, which usually undergo quality appraisal before they are included in an HTA analysis. Before deciding to use expert opinion, authors should become clear about what the risks of using expert data are and how to avoid them. Crucial points for the use of expert data in HTA are to make the process transparent, and to minimize, quantify, and describe uncertainties around expert data (8).

Existing research about expert elicitation in HTA and related fields comes mainly from decision-analytic modeling and clinical guideline development. The former combines various methods, while the latter mainly applies consensus panels as expert opinion. Guideline development also deals with methods to formalize the integration of subjective judgments (e.g., GRADE) (51); however, the scope and procedure of guideline development differs from that of HTA and transferability of its methods is limited. Many established methods come from social research and are also applied in other fields than healthcare. Looking at the nonmedical literature, we see that for example ecology, environmental research, operations research or risk analysis, are confronted with similar problems in their specific form of technology assessment, mainly regarding elicitation of quantitative data. Bayesian methods, for example, are used in different disciplines and allow for incorporation of expert opinion regarding prior distributions of parameter estimates (52).

The Delphi method is probably the most common technique for elicitation from groups of experts (53). It is characterized by anonymous answers with feedback over one or more rounds. However, it is sometimes used as a catch-all term for different kinds of consensus groups (8). Sullivan and Payne (8) suggests to better elicit single expert data for parameter values and aggregate them mathematically instead of applying consensus methods.
Evans provides a hierarchy of expert data, reflecting for which categories experts are more likely to give credible and correct estimates (9). Model validation and information on treatment patterns were judged more reliable than costs and probability estimates. A survey among twenty-four HTA agencies found that 75 percent used expert opinion in the majority or all of their HTAs to amend and interpret trial data (Douw 2008, HTAi, conference abstract). The agencies mainly obtained qualitative data (70 percent) through informal conversation (61 percent). This indicates that expert knowledge data like cost and probability estimates are not the primary goal of expert consultation. However, the proportion of quantitative estimates might increase due to the rising number of modeling studies.

\section{CONCLUSION}

The issuers of publicly funded HTA reports consider expert opinion as an information source, but prevailingly do not give sufficient guidance for elicitation in their handbooks. HTA doers should be aware of the uncertainty surrounding expert opinion and the influence of the elicitation design. Further research should focus on how expert elicitation is actually performed in HTA and on how to transfer established methods from other fields into HTA.

\section{Policy Implications}

HTA Agencies should integrate existing knowledge on expert elicitation methods from all related fields into their guiding documents to formalize the use of expert data and make the process more transparent, systematic, and reliable. Particularly when experts provide quantitative data, explicit recommendations should be given.

\section{SUPPLEMENTARY MATERIAL}

\begin{tabular}{|c|c|c|c|}
\hline Supplementary & able & 1: & http://dx.doi.org/10.1017/ \\
\hline S026646231600020 & & & \\
\hline $\begin{array}{l}\text { Supplementary } \\
\text { S026646231600020 }\end{array}$ & Table & 2: & http://dx.doi.org/10.1017/ \\
\hline
\end{tabular}


Supplementary Table 3: http://dx.doi.org/10.1017/ S0266462316000209

Supplementary Figure 1: http://dx.doi.org/10.1017/ S0266462316000209

\section{CONFLICTS OF INTEREST}

T.H. received grants from the Tiroler Wissenschaftsfonds (Tyrolian Science Fund), grant number GZ: UNI - 4040/1009. The other authors have nothing to disclose.

\section{REFERENCES}

1. Busse R, Orvain J, Valesco M, et al. Best practice in undertaking and reporting health technology assessments. Int J Technol Assess Health Care. 2002;18:361-422.

2. Velasco-Garrido M, Busse R. Health technology assessment. An introduction to objectives, role of evidence, and structure in Europe. Geneva: WHO; 2005.

3. LBI. (Internes) Manual Abläufe und Methoden. Wien: Ludwig Boltzmann Institut für Health Technology Assessment (LBI-HTA); 2008.

4. Drummond MF, Schwartz JS, Jonsson B, et al. Key principles for the improved conduct of health technology assessments for resource allocation decisions. Int $J$ Technol Assess Health Care. 2008;24:244-258; discussion 362-268.

5. OCEBM Levels of Evidence Working Group. The Oxford Levels of Evidence 2: Oxford Centre for Evidence-Based Medicine; 2011. http: $/ /$ www.cebm.net/index.aspx?o=5653 (accessed June 6, 2014).

6. Fleischner B, Mittermayer T, Wild C. Beschaffungsprozesse ausgewählter Produktgruppen in Krankenanstalten -Orthopädische und kardiologische Implantate Wien: Ludwig Bolzmann Institut; 2009.

7. Siebert U, Sroczynski G, Aidelsburger P, et al. Clinical effectiveness and cost-effectiveness of tailoring chronic hepatitis $\mathrm{C}$ treatment with peginterferon alpha-2b plus ribavirin to $\mathrm{HCV}$ genotype and early viral response: A decision analysis on new German guidelines. Pharmacoeconomics. 2009;27:341-354.

8. Sullivan W, Payne K. The appropriate elicitation of expert opinion in economic models: Making expert data fit for purpose. Pharmacoeconomics. 2011;29:455-459.

9. Evans C, Crawford B. Expert judgement in pharmacoeconomic studies. Guidance and future use. Pharmacoeconomics. 2000;17:545-553.

10. EUnetHTA. Methods for health economic evaluations - A guideline based on current practices in Europe. Copenhagen: European network for Health Technology Assessment; 2015.

11. NICE. Interventional procedures programme process guide. London: National Institute for Health and Clinical Excellence (NICE); 2009.

12. NICE. Interventional procedures programme methods guide. London: National Institute for Health and Clinical Excellence; 2007.

13. NICE. Guide to the methods of technology appraisal. London: National Institute of Health and Excellence; 2013.

14. NICE. Guide to the processes of technology appraisal. London: National Institute for Health and Care Excellence; 2014.

15. CRD. Systematic Reviews. CRD's guidance for undertaking reviews in health care. York: Centre for Reviews and Dissemination (CRD); 2009.

16. IQWiG. Allgemeine Methoden 4.2. Köln: Institut für Qualität und Wirtschaftlichkeit im Gesundheitswesen [Institute for Quality and Efficiency in Health Care], 2015.

17. ASERNIP-S. ASERNIP-S systematic review process. North Adelaide: Australian Safety and Efficacy Register of New Interventional Procedures-Surgical (ASERNIP-S); 2003.
18. HAS. Rapid assessment method for assessing medical and surgical procedures. Haute Autorité de Santé; 2007.

19. NICE. Medical technologies evaluation programme methods guide. London: National Institute for Health and Clinical Excellence, 2011.

20. HAS. Assessment of ethical aspects. Saint-Denis La Plaine: Haute Autorité de Santé; 2013.

21. AAZ. The Croatian guideline for health technology assessment process and reporting. Zagreb: Agency for Quality and Accreditation in Health Care, 2011.

22. DACEHTA. Health technology assessment handbook. Copenhagen: Danish Centre for Health Technology Assessment, National Board of Health (DACEHTA); 2007.

23. LBI. (Externes) Manual. Selbstverständnis und Arbeitsweise. Wien: Ludwig Boltzmann Institut fur Health Technology Assessment (LBIHTA), 2007.

24. OHTAC. Advancing health. Evidence-based advice on health technology. Ontario: Ontario Health Technology Advisory Committee (OHTAC); 2009.

25. NICE. Medical technologies evaluation programme process guide. London: National Institute for Health and Clinical Excellence; 2011.

26. Cleemput I, Neyt M, Van de Sande S, Thiry N. Belgian guidelines for economic evaluations and budget impact analyses: 2nd ed. Brussels: Belgian Health Care Knowledge Centre (KCE); 2012.

27. EuroScan. A toolkit for the identification and assessment of new and emerging health technologies. Birmingham: EuroScan International Network; 2014.

28. HIQA. Guidelines for the budget impact analysis of health technologies in Ireland. Dublin: Health Information and Quality Authority (HIQA); 2014.

29. CVZ. Assessment of established medical science and medical practice. Dieman: College voor zorgverzekeringen. 2007.

30. Fröschl B, Bornschein B, Brunner-Ziegler S, et al. Methodenhandbuch für Health Technology Assessment. Wien: Gesundheit Österreich, 2012.

31. HIQA. Guidelines for stakeholder engagement in health technology assessment in Ireland. Dublin: Health Information and Quality Authority; 2014.

32. Pertl D, Fröschl B, Rosian-Schikuta I, Schtürzlinger H. Prozesshandbuch für Health Technology Assessment. Vienna: Gesundheit Österreich (GÖG); 2010.

33. HAS. Choices in methods for economic evaluation. Saint-Denis La Plaine: Haute Autorité de santé; 2012.

34. PHARMAC. Prescription for pharmacoeconomic analysis. Methods for cost-utility analysis. Wellington: Pharmaceutical Management Agency; 2012.

35. Swartenbroekx N, Obyn C, Guillaume P, Lona M, Cleemput I. Manual for cost-based pricing of hospital interventions. Brussels: Belgian Health Care Knowledge Centre (KCE), 2012.

36. CADTH. Guidelines for the economic evaluation of health technologies. Ottawa, Canada: Canadian Agency for Drugs and Technologies in Health (CADTH); 2006.

37. Mittmann N, Evans WK, Rocchi A, et al. Addendum to CADTH's Guidelines for the economic evaluation of health technologies: Specific guidance for oncology products. Ottawa, Canada: Canadian Agency for Drugs and Technologies in Health (CADTH); 2009.

38. MOH. Pharmacoeconomic guideline for Malaysia. Putrajaya: Ministry of Health Malaysia; 2012.

39. HIQA. Guidelines for the economic evaluation of health technologies in Ireland. Dublin: Health Information and Quality Authority; 2010.

40. CVZ. Guidelines for pharmacoeconomic research, updated version. Dieman: College voor zorgverzekeringen; 2006. 
41. AHTAPol. Guidelines for conducting health technology assessment. Warsaw: Agency for Health Technology Assessment in Poland (AHTAPol); 2009.

42. Alves da Silva E, Gouveia Pinto C, António Pereira J, Drummond M, Trindade R. Guidelines for economic drug evaluation studies. Portugal: National Authority of Medicines and Health Products (INFARMED); 1998.

43. HIQA. Guidelines for evaluating the clinical effectiveness of health technologies in Ireland. Dublin: Health Information and Quality Authority; 2014.

44. INAHTA. INAHTA's Working Group on Handling Ethical Issues. Alberta, Canada: International Network of Agencies for Heath Technology Assessment (INAHTA) Ethics Working Group; 2005.

45. OHTAC. Decision determinants guidance document. Ontario: The Ontario Health Technology Advisory Committee (OHTAC); 2010.

46. Cleemput I, Neyt M, Thiry N, DeLaet C, Leys M. Threshold values for cost-effectiveness in health care. Brussels: The Belgian Health Care Knowledge Centre (KCE), 2008.

47. HAS. General methods for assessing health technologies. Saint-Denis, France: Haute Autorité de Santé; 2007.

48. DAHTA@DIMDI. Handbuch für Autoren zur Erstellung von HTABerichten. Köln: Deutsche Agentur für Health Technology Assessment des Deutschen Instituts für Medizinische Dokumentation und Information; 2008.

49. HIQA. Guidelines for the retrieval and interpretation of economic evaluations of health technologies in Ireland. Dublin: Health Information and Quality Authority; 2014.

50. Grigore B, Peters J, Hyde C, Stein K. Methods to elicit probability distributions from experts: A systematic review of reported practice in health technology assessment. Pharmacoeconomics. 2013;31:9911003.

51. Onate-Ocana LF, Ochoa-Carrillo FJ. The GRADE system for classification of the level of evidence and grade of recommendations in clinical guideline reports. Cir Cir. 2009;77:417-419.

52. Ades AE, Sculpher M, Sutton A, et al. Bayesian methods for evidence synthesis in cost-effectiveness analysis. Pharmacoeconomics. 2006;24:1-19.

53. Brown B. Delphi Process: A methodology used for the elicitation of opinions of experts. Santa Monica, CA: The RAND Corporation; 1968.

54. EUnetHTA. The HTA core model. Version 2.0. Copenhagen: European network for Health Technology Assessment, 2013.

55. NICE. Diagnostics assessment programme manual. London: National Institute for Health and Clinical Excellence; 2011. 